\title{
Negative feedback within a mutualism: host-specific growth of mycorrhizal fungi reduces plant benefit
}

\author{
James D. Bever \\ Department of Biology, Indiana University, Bloomington, IN 47405, USA (jbever@indiana.edu)
}

\begin{abstract}
A basic tenet of ecology is that negative feedback on abundance plays an important part in the coexistence of species within guilds. Mutualistic interactions generate positive feedbacks on abundance and therefore are not thought to contribute to the maintenance of diversity. Here, I report evidence of negative feedback on plant growth through changes in the composition of their mutualistic fungal symbionts, arbuscular mycorrhizal (AM) fungi. Negative feedback results from asymmetries in the delivery of benefit between plant and AM fungal species in which the AM fungus that grows best with the plant Plantago lanceolata is a poor growth promoter for Plantago. Growth of Plantago is, instead, best promoted by the AM fungal species that accumulate with a second plant species, Panicum sphaerocarpon. The resulting community dynamic leads to a decline in mutualistic benefit received by Plantago, and can contribute to the coexistence of these two competing plant species.
\end{abstract}

Keywords: mutualism; mycorrhizae; maintenance of diversity; negative feedback; coexistence; plant-microbe interactions

\section{INTRODUCTION}

A basic tenet of ecology is that negative feedback on abundance (i.e. negative density dependence) plays an important part in the regulation of population density and, therefore, in the coexistence of species within guilds (Levins 1974; May 1974). This negative feedback has generally been thought to result from antagonistic interactions such as competition, predation and parasitism (Tilman \& Pacala 1993; Chesson 2000). Mutualistic interactions in which both participants benefit from their association, while known to be common (Boucher 1985), have not been thought to generate negative feedback and therefore were not expected to contribute to the coexistence of species (May 1974). While mutualism can be defined as a positive feedback process in density, it is possible that the community dynamics between mutualistically interacting guilds of species can generate net negative feedback on the relative abundance of species within these guilds (Bever 1999). In this study, I use a series of manipulative experiments to demonstrate negative feedback on plant growth through changes in the composition of their mutualistic fungal symbionts, arbuscular mycorrhizal (AM) fungi. This negative feedback results from the asymmetry in the delivery of benefit between plant and mycorrhizal fungal species.

AM fungi can facilitate plant growth by increasing access to soil resources. The interaction is a well-established mutualism, as the fungus, in turn, is wholly dependent on carbohydrates from the plant. The ecological importance of this interaction is broadly appreciated, as most plant species, including most agricultural plants, associate with AM fungi (see Smith \& Read 1997). While the association is thought to be largely non-specific, there is growing evidence of host-specific differences in plant response to fungi and in fungal response to plants. The extent of plant growth promotion by AM fungi depends upon the specific plant and fungal combinations (see Streitwolf-Engel et al. 1997; van der Heijden et al. 1998). Conversely, the growth response of AM fungal species also depends on the associated host-plant species (see Johnson et al. 1992; Bever et al. 1996; Eom et al. 2000).

This mutual interdependence of plant and AM fungal population growth rates could generate complex community dynamics between the plant and fungal guilds. Host-specific changes in the AM fungal community could lead to increases in the relative growth rates of the most abundant plant (i.e. positive feedback) or to decreases in the relative growth rates of the most abundant plant species (i.e. negative feedback) (Bever et al. 1997; Bever 1999). These two dynamics lead to very different predictions for the community. Positive feedback causes a strengthening of the mutualism between plant and fungal guilds, but a decline in species diversity. Negative feedback causes a weakening of the mutualism, but can contribute to the coexistence of competing plant species (Bever 1999).

Currently, there is little evidence of such complex community feedback dynamics between plants and fungi, or within any other mutualism. Several studies have identified patterns consistent with negative feedback through the AM fungal community (Johnson et al. 1991; Bever 1994; Kiers et al. 2000). However, in each case, accumulation of host-specific root pathogens provides a likely alternative mechanism (Mills \& Bever 1998; Bever 2002). An additional study indicates a decline in crop yield due to the accumulation of an AM fungal species (Modjo \& Hendrix 1986), but the accumulation of this fungus was probably driven as much from the fertilization treatments as from host-specificity of fungal growth rates. Experimental tests of plant and fungal growth rates using pure cultures have indicated highly asymmetric relative growth rates between plants and $\mathrm{AM}$ fungi that would generate negative feedback (Pringle 2001; Bever et al. 2002). 


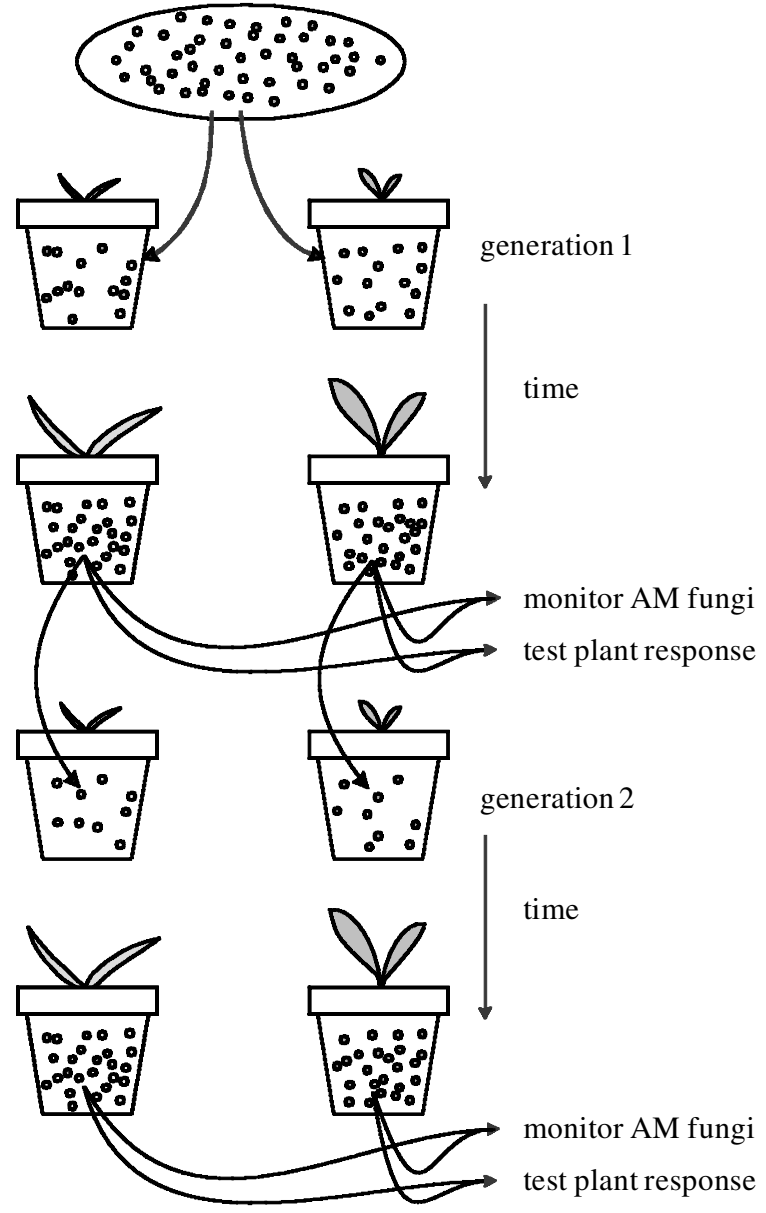

Figure 1. Design of feedback experiment. The initial AM fungal community was created by mixing equal volumes of pure cultures of eight fungal species. This community was then grown with four host species (two are shown) for a five month growing season (generation 1). The fungal communities were then harvested, assayed, and then reinoculated onto their same plant host species at the start of the second growing season (generation 2). At the end of each growing season, the composition of the AM fungal community was monitored and the fungal communities were used as inoculum in a separate test experiment that evaluated the growth response of the plant species in association with each of the fungal communities.

Predictions of fungal dynamics in this study, however, are dependent upon counts of spores, an incomplete measure of AM fungal population growth rate.

Evaluating the nature of the plant-AM fungal community dynamics is complicated by the difficulties in measuring relative rates of population growth of AM fungi. AM fungi can reproduce vegetatively through hyphal extension and by the production of resting propagules in the form of asexual spores. The hyphal structures, which are embedded in the root and soil, cannot be identified morphologically and are hard to quantify. Monitoring of the AM fungal community composition therefore often depends on counts of spores, an imperfect measure. In this study, I circumvented the difficulties in measurement of AM fungal population growth rates by manipulating and monitoring replicate laboratory AM fungal communities (figure 1). Follow-up tests indicated negative feedback through the soil community and the AM fungal causal mechanism was confirmed in an independent assay of plant growth response.

\section{METHODS}

Two complementary approaches were used to test negative feedback through the AM fungal community. First, I manipulated and evaluated AM fungal communities that were constructed by mixing pure cultures of individual AM fungal species. Specifically, replicate AM fungal communities were grown on individual host plants for a growing season, during which time the composition of the fungal community may have differentiated due to host-specific differences in fungal population growth rates (figure 1). These fungal communities were then used as inoculum in a separate test experiment evaluating plant growth response. The inoculum in this test experiment includes both the spores and hyphal structures of AM fungi and therefore accurately represents the entire AM fungal community. The second approach confirms the AM fungal mechanism of negative feedback by monitoring the host-specific differentiation of the AM fungal community during the feedback experiment (figure 1). The causal relationship between the feedback on plant growth and the changes in the AM fungal community composition was then tested by an independent assessment of plant growth in response to inoculation with the original pure cultures of AM fungi.

\section{(a) Study system}

Four plant species and eight AM fungal species were used in this study, all of which were isolated from within a $75 \mathrm{~m}^{2}$ area of a North Carolina grassland (Bever et al. 1996, 2001). The four plant species included two herbs, Allium vineale and Plantago lanceolata and two grasses, Anthoxanthum odoratum and Panicum sphaerocarpon. Six genotypes of each of the plant species used in this study were clonally propagated. Anthoxanthum and Panicum were cloned and cleaned as previously described (Bever 1994). Clones of Allium were replicated with their asexually produced bulbils, and clones of Plantago were grown from leaf cuttings dipped in indoleacetic acid. All fungal cultures were started from a single spore and maintained in isolation in a growth chamber within the Duke University Phytotron to avoid contamination by soil pathogens. These eight pure cultures were mixed in equal proportion to create the initial AM fungal communities used for the feedback experiments. The eight AM fungal species were: Acaulospora morrowiae, Archaeospora trappei, Gigaspora gigantea, Gi. decipiens, Scutellospora calospora, S. pellucida, S. reticulata and an undescribed species of Glomus (identified as Gl. D3 (Bever et al. 1996)). Each of these species has been deposited in an international culture collection (INVAM) as previously described (Bever et al. 1996). Cultures were propagated on a common host, Sorghum vulgare, and grown in a growth chamber for four and a half months at which time there was abundant sporulation. The cultures were then stored dry for several months in the refrigerator to break the dormancy of the spores prior to use.

\section{(b) AM fungal community training experiment}

Replicate $600 \mathrm{cc}$ pots were filled with $400 \mathrm{cc} 1: 1$ mixture of sterile soil and sand mix and inoculated with $200 \mathrm{cc}$ of the initial mixture of pure cultures of eight AM fungal species. Into these pots were planted soil-microbe free plants of one of six geno- 
types of each of the four plant species (three replicates of each of the six genotypes of each of the four plant species). The plants were grown in a growth chamber with conditions set to mimic Spring in North Carolina ( $12 \mathrm{~h}$ day length; $27^{\circ} \mathrm{C}$ during the day and $20^{\circ} \mathrm{C}$ during the night). After four months, when the perennial plants began to die back, watering was reduced and AM fungi sporulated (Bever et al. 1996). These soils were stored dry in the refrigerator for several months before use to break the dormancy of the spores. The second generation of the experiment was started as the first with each AM fungal community being grown with its same plant genotype. The composition of the AM fungal community within each pot was monitored as previously described (Bever et al. 1996). Host-specific differentiation of AM fungal communities was evaluated using multivariate profile analysis on ranks of spore densities as previously detailed (Bever et al. 1996).

\section{(c) Tests of feedback on plant growth}

To test for feedback, the effect of the differentiated AM fungal communities on plant growth was evaluated by growing replicates of the six genotypes of each plant species with individual fungal communities. In the test experiment for generation 1, each plant genotype was grown in an uninoculated control and with the AM fungal community from its own genotype, from a second genotype of its own species and from one genotype of each of the other species. This design was repeated for two replicate cultures for each genotype. The test experiment after the second generation focused on the Panicum-Plantago comparison. The design was as in generation 1, with balanced replication of each species in their own and each other's fungal communities. In both test experiments, soil-microbe free plants were planted into $400 \mathrm{cc}$ pots of sterile soil and sand mix inoculated with $50 \mathrm{cc}$ of inoculum from the cultures. They were grown in growth chambers as above for 10 weeks after which they were harvested, dried and weighed. Feedback was tested using a series of pairwise home versus away contrasts as previously derived (Bever et al. 1997).

\section{(d) Plant growth response to AM fungal isolates}

To confirm the mycorrhizal mechanism of the feedback between Panicum and Plantago, I evaluated the growth response of these species to inoculation with three AM fungal species that showed host-specific growth rates. Panicum and Plantago seedlings were grown in $500 \mathrm{cc}$ sterile soil and sand mix $(1: 1 \mathrm{v} / \mathrm{v})$ with seven inoculation treatments: the three AM fungal species, a separate control treatment for each AM fungal inoculum, and a fourth un-amended control. AM fungal treatments received $50 \mathrm{cc}$ of inoculum. The controls for each AM fungal species received equal amounts of heat-pasteurized inoculum plus a drench of non-AM fungal filtrate from the inoculum, thereby controlling for potential mineral and non-mycorrhizal microbial components of the inoculum. AM fungal treatments, microbial controls and the sterile control were replicated 11,9 and 7 times, respectively. The plants were grown and harvested as in the previous growth experiments. Microscopic examination of roots confirmed infection of mycorrhizal treatments and the absence of mycorrhizae in the controls. There were no statistical differences in plant growth between the four controls. Therefore, the differences in growth between inoculated and control treatments can be attributed to the different AM fungal species.

\section{RESULTS}

\section{(a) Tests of feedback on plant growth}

After the first generation of training, the plants generally grew better with each other's AM fungal community than with their own $\left(F_{1,236}=9.0, p<0.003\right)$. This overall effect was due to significant negative feedback between two of the six pairs of plant species. After adjusting for multiple comparisons, there was weak evidence for negative feedback between Allium and Panicum $\left(F_{1,236}=6.03, p<0.05\right)$ and strong evidence for negative feedback between Pan$i$ cum and Plantago $\left(F_{1,236}=17.04, p<0.0001\right)$. With this strong evidence of negative feedback between Panicum and Plantago, I focused the rest of this study on these two plant species.

In specifically comparing Plantago and Panicum, Plantago was more responsive than Panicum to AM fungal inoculation in general, and was specifically more responsive to the AM fungal community developed in association with Panicum relative to that developed with itself (figure $2 a$ ). These two patterns of the greater responsiveness of Plantago to inoculation and the negative feedback between Plantago and Panicum were also significant after the second generation of training (figure $2 b$ ).

\section{(b) Host-specificity of AM fungal population growth rates}

I found strong evidence of host-specific differences in the AM fungal population growth rates. These results have been previously described in full (Bever 2002). Here, I present analyses of the differentiation between Panicum and Plantago. While the total biovolume of AM fungal spores produced in either generation was not significantly different between Panicum and Plantago, the AM fungal community composition was highly divergent. For both hosts, the biovolume was largely made up of four AM fungal species: Ac. morrowiae, Ar. trappei, Gi. gigantea and $S$. calospora. Three of these species showed strong differences in growth with the two hosts. The fungi Ac. morrowiae and Ar. trappei sporulated more strongly with Panicum than Plantago, while S. calospora sporulated more strongly in association with Plantago than Panicum (figure 3).

While it is possible that these differences in sporulation rates reflect host-specific differences in fungal phenology, two lines of statistical evidence strongly indicate the existence of underlying host-specificity in AM fungal population growth rates. First, host-specific divergence between the AM fungal communities became stronger through time as tested by the species effect within multivariate profile analysis (generation 1: Wilks' $\lambda F_{9,151}=8.97, p<0.0001$; generation 2: Wilks' $\left.\lambda F_{3,148}=14.78, p<0.0001\right)$. Second, for each fungal species, when spore abundance in the first generation is included as a covariate predictor of spore abundance in the second year, both spore abundance in year 1 and host in year 2 are significant predictors of spore abundance in the second generation. Both of these analyses indicate that spore density for these species accurately represents their relative population growth rates and therefore the host-specific differences in sporulation that we measured reflect underlying host-specific differences in AM fungal population growth rates. 
(a)

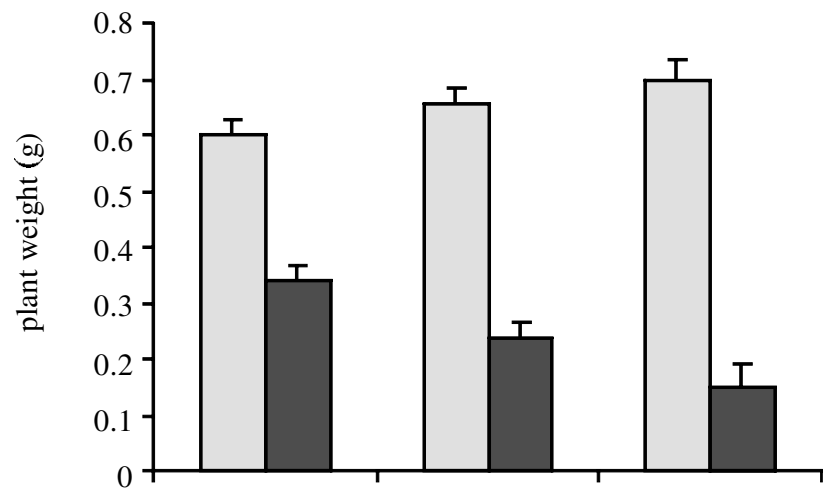

(b)

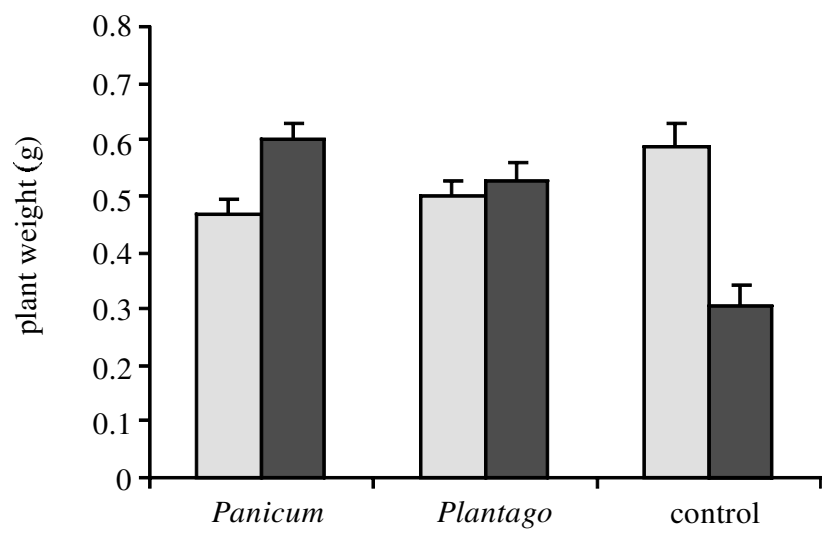

AM fungal community

Figure 2. Plant growth responses to AM fungal communities in (a) generation 1 and (b) generation 2. Grey bars represent Panicum and black bars Plantago. Panicum and Plantago responded differently to their AM fungal communities. Plantago was more responsive than Panicum to AM fungal inoculation in general (generation $1: F_{1,78}=30.13$, $p<0.0001$; generation $2: F_{1,96}=36.16, p<0.0001$ ). Plantago was particularly responsive to the fungal community from Panicum as tested by a home versus away contrast (Bever et al. 1997) (generation 1: $F_{1,78}=17.04, p<0.0001$; generation 2: $\left.F_{1,96}=3.75, p=0.05\right)$. Statistics were performed on square-root transformed values of total plant biomass.

\section{(c) Plant growth response to individual AM fungal species}

Given the host-specific differentiation observed in the AM fungal communities (figure 3), the negative feedback on Plantago growth could result from differential responses of Panicum and Plantago to association with Ac. morrowiae, Ar. trappei or S. calospora. This interpretation was supported in independent evaluation of Panicum and Plantago growth responses to inoculation by these three AM fungal species. Plantago, again, was more responsive to AM fungal inoculation than Panicum. Most importantly, Plantago was particularly responsive to inoculation with Ac. morrowiae and Ar. trappei relative to $S$. calospora, while Panicum grew better with $S$. calospora than with Ac. morrowiae and Ar. trappei (figure 4). Therefore, the independent test of plant response to these AM fungal isolates corroborates the AM fungal mechanism for the observed negative feedback on plant growth (figure 2). (a)

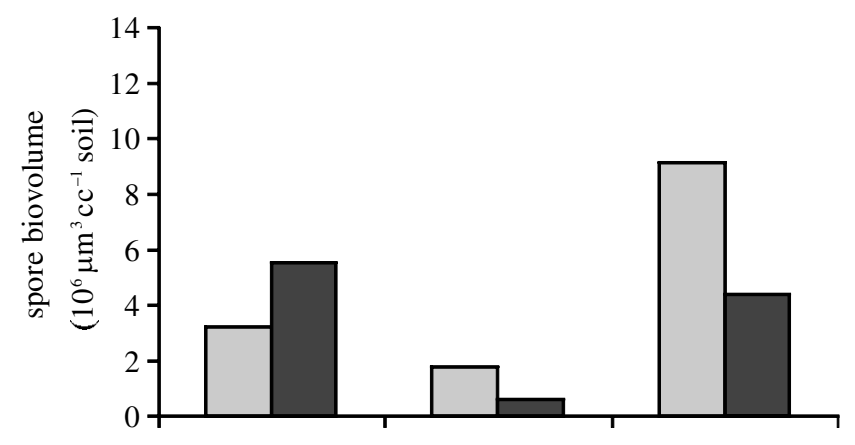

(b)

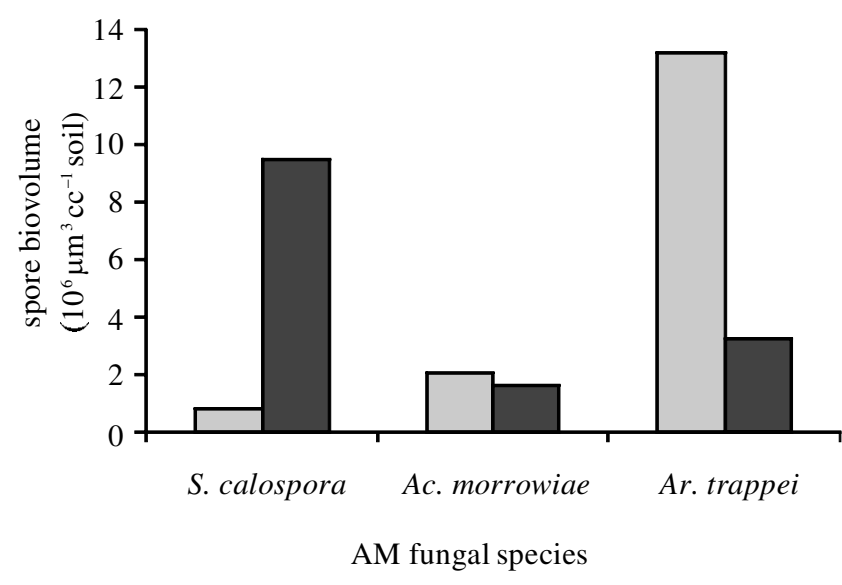

Figure 3. AM fungal spore production in (a) generation 1 and $(b)$ generation 2. Grey bars represent Panicum and black bars Plantago. The AM fungal communities differentiated on Panicum and Plantago. Scutellospora calospora grew better with Plantago (generation 1: $F_{1,19}=3.06, p<0.1$; generation 2: $\left.F_{1,19}=15.95, p<0.001\right)$. Acaulospora morrowiae and Archaeospora trappei grew better with Panicum (for Ac. morrowiae, generation 1: $F_{1,19}=50.8, p<0.0001$; generation 2: not significant; for Ar. trappei, generation 1: $F_{1,19}=16.17, p<0.001$; generation $2: F_{1,19}=15.36$, $p<0.001)$. Mean spore biovolume was calculated from the average spore production weighted by the average spore volume and statistical tests were on ranks of spore abundance.

\section{DISCUSSION}

Negative feedback through changes in the whole soil community has been observed in previous studies of Plantago and Panicum (Bever et al. 1997). Previous work on soil pathogens with these two species has suggested that accumulation of soil pathogens in the genus Pythium that are specific to Panicum could contribute to the negative feedback (Mills \& Bever 1998). The present study supports the operation of a second, complementary soil microbial mechanism of negative feedback between these two species: host-specific changes in the composition of the AM fungal community. Three lines of evidence come together to support the interpretation of a mycorrhizal cause of the negative feedback. First, great effort was put into excluding soil pathogens from our experimental system by starting all AM fungal cultures from single spores and then maintaining these cultures and the experimental communities away from sources of soil pathogens. Even with these efforts, a possibility of contamination of the cul- 


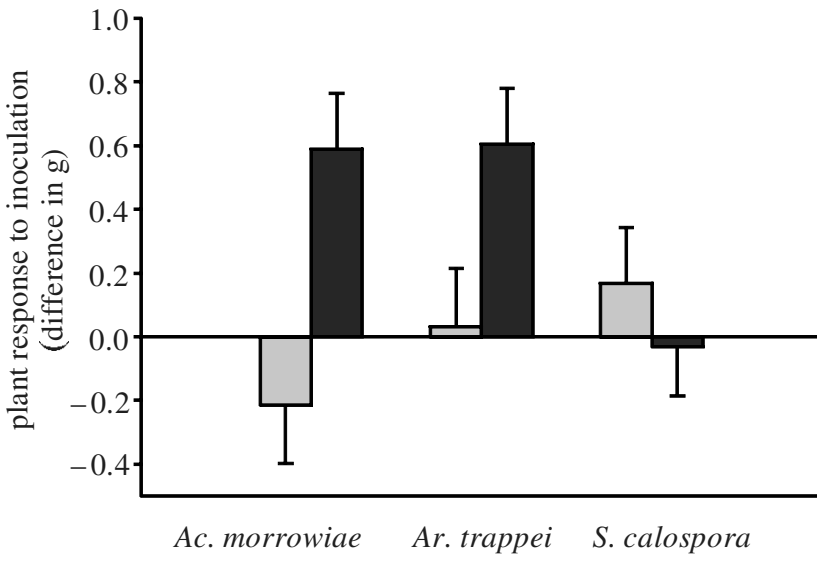

AM fungal species

Figure 4. Plant growth response to individual AM fungal species. Grey bars represent Panicum and black bars Plantago. Differences in mean plant biomass between inoculations and controls are presented. Plantago was more responsive to AM fungal inoculation than Panicum $\left(F_{1,109}=5.33, p=0.02\right)$. Plantago was particularly responsive to inoculation with Acaulospora morrowiae and Archaeospora trappei relative to Scutellospora calospora $\left(F_{1,109}=5.45\right.$, $p=0.02)$. Statistics were performed on square-root transformed values.

tures remains. However, no evidence of root pathogens was observed in the root system of the test experiments. Moreover, the plant growth responses to the individual AM fungal species (figure 4), when combined with the differentiation observed in the AM fungal community (figure 3), can explain the negative feedback observed in the test experiment (figure 2). With the internal consistency of these experiments, it is unnecessary and non-parsimonious to invoke the action of any undetected pathogens to explain the negative feedback observed in the test experiment (figure 2). The possibility remains that microorganisms that have been observed living within spores of AM fungi (see Walley \& Germida 1996; Redecker et al. 1999) could contribute to the impact of these fungi on plant growth. However, for these microorganisms to play a part in the observed negative feedback, they would have to be stably inherited with individual AM fungal species to account for the internal consistency of these results. In such a case, the AM fungal species and its symbionts would be behaving as a single organism.

The decreased benefit that Plantago receives from its own AM fungal community relative to that of Panicum appears to result from an asymmetry in delivery of benefit between plants and AM fungi. While $S$. calospora has its highest population growth rate on Plantago, Plantago does not benefit from this fungus as much as it benefits from Ac. morrowiae and Ar. trappei, which themselves accumulate with Panicum (figure 5). The resulting dynamic may contribute to plant species coexistence. As Plantago becomes increasingly common, S. calospora would be predicted to increase, causing Plantago's growth rate to decrease and allowing Panicum to increase. Conversely, a high abundance of Panicum would be predicted to have an indirect beneficial effect on the growth of Plantago

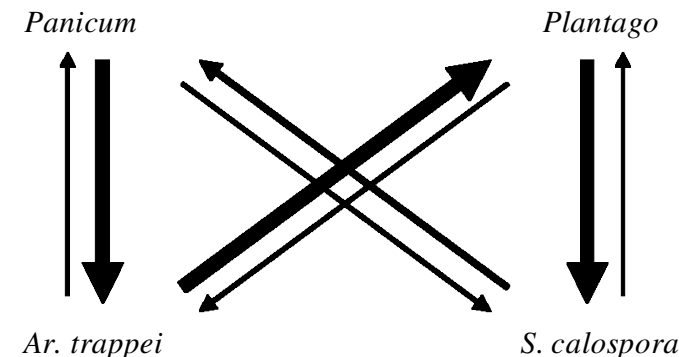

Ac. morrowiae

Figure 5. Asymmetric fitness relationships between plants and AM fungi generate negative feedback. The arrows indicate the direction of benefit and the thickness of the arrows indicates the relative magnitude of benefit. The presence of Panicum increases the relative abundance of Acaulospora morrowiae and Archaeospora trappei, thereby increasing the relative performance of Plantago.

through the changes in the AM fungal community. To my knowledge, this is the first demonstration of negative feedback being generated within a mutualism, and this result adds credence to previous observations indicating similar dynamics (Johnson et al. 1991; Bever 1994; Kiers et al. 2000; Bever et al. 2002).

It is interesting to note that Panicum is a native species to North Carolina while Plantago is exotic. In this context, the present study indicates that the presence of the native plant, Panicum, indirectly facilitates the establishment of the exotic plant species, Plantago, through changes in the composition of the AM fungal community. Mycorrhizal facilitation of invasive plant species has been suggested in several other studies (Marler et al. 1999; Callaway et al. 2001; Klironomos 2002). The AM fungal community dynamics observed in the present study could specifically contribute to an explanation of mycorrhizal mediation of competitive superiority of an invasive forb species against a native perennial grass species in the western USA (Marler et al. 1999; Callaway et al. 2001).

The observation of negative feedback through changes in the AM fungal community between Panicum and Plantago in the present study does not indicate that all feedback through the mycorrhizal fungal community should be negative. Indeed, we have circumstantial evidence of a positive feedback dynamic between other plant and AM fungal species within the same community (Bever et al. 2001). Rather, these results with Panicum and Plantago broaden our understanding of the range of dynamics possible within a mutualism and lead to questions of how often, and under what conditions, do dynamics between plants and AM fungi result in positive versus negative feedback. These questions are of importance beyond the plant-AM fungal interaction, as they are equally relevant to the dynamics of other mutualisms (Bronstein 1994). Moreover, as the benefit that Plantago receives from its AM fungal community degrades over time, a microevolutionary process of this type could lead to the dissolution of the mutualism, as has been found to occur within several mutualisms over evolutionary time (Fitter \& Moyersoen 1996; Hibbett et al. 2000; Lutzoni et al. 2001). Therefore, identifying what conditions lead to positive versus negative feedback represents an important step 
towards understanding the evolutionary maintenance of mutualism.

The present study uses a series of experimental manipulations to illustrate the complex dynamics possible within the plant-AM fungal mutualism. The results of this study indicate that dynamics within the AM fungal community can contribute to the maintenance of diversity within plant communities. While the generality of this mechanism awaits further experimentation, a positive effect of AM fungal diversity on plant diversity has recently been demonstrated in two grassland systems (van der Heijden et al. 1998). Together with other recent demonstrations of microbial control over plant community diversity and dynamics (van der Putten et al. 1993; Mills \& Bever 1998; Clay \& Holah 1999; Packer \& Clay 2000; Callaway et al. 2001; Klironomos 2002), these studies call for heightened attention to the potential importance of microbes in community processes. These studies also raise the concern that loss of soil microbial diversity due to agriculture (Helgason et al. 1998; Douds \& Millner 1999) and other anthropogenic disturbance (Egerton-Warburton \& Allen 2000) may have unanticipated negative consequences on community functions.

I thank J. Morton, J. Antonovics, P. Schultz, A. Pringle, M. Watson, K. Clay, E. Brodie, A. Agrawal, A. Bennett, S. Richardson, K. Mills and B. Chaudhary for advice and assistance. This project was supported by grants from USDA and NSF.

\section{REFERENCES}

Bever, J. D. 1994 Feedback between plants and their soil communities in an old field community. Ecology 75, 1965-1977.

Bever, J. D. 1999 Dynamics within mutualism and the maintenance of diversity: inference from a model of interguild frequency dependence. Ecol. Lett. 2, 52-61.

Bever, J. D. 2002 Host-specificity of AM fungal population growth rates can generate feedback on plant growth. Plant and Soil 244, 281-290.

Bever, J. D., Morton, J. B., Antonovics, J. \& Schultz, P. A. 1996 Host-dependent sporulation and species diversity of arbuscular mycorrhizal fungi in a mown grassland. F. Ecol. 84, 71-82.

Bever, J. D., Westover, K. M. \& Antonovics, J. 1997 Incorporating the soil community into plant population dynamics: the utility of the feedback approach. F. Ecol. 85, 561-573.

Bever, J. D., Schultz, P. A., Pringle, A. \& Morton, J. B. 2001 Arbuscular mycorrhizal fungi: more diverse than meets the eye, and the ecological tale of why. Bioscience 51, 923-931.

Bever, J. D., Pringle, A. \& Schultz, P. A. 2002 Dynamics within the plant-AM fungal mutualism: testing the nature of community feedback. In Mycorhizal ecology (ed. M. G. A. van der Heijden \& I. Sanders), pp. 267-292. Berlin: Springer.

Boucher, D. H. 1985 The biology of mutualism. New York: Oxford University Press.

Bronstein, J. L. 1994 Our current understanding of mutualism. Q. Rev. Biol. 69, 31-51.

Callaway, R. M., Newingham, B., Zabinski, C. A. \& Mahall, B. E. 2001 Compensatory growth and competitive ability of an invasive weed are enhanced by soil fungi. Ecol. Lett. 4, 429-433.

Chesson, P. 2000 Mechanisms of maintenance of species diversity. A. Rev. Ecol. Systems 31, 343-366.

Clay, K. \& Holah, J. 1999 Fungal endophyte symbiosis and plant diversity in successional fields. Science 285, 17421744 .

Douds, D. D. \& Millner, P. D. 1999 Biodiversity of arbuscular mycorrhizal fungi in agroecosystems. Agricult. Ecosyst. Environ. 74, 77-93.

Egerton-Warburton, L. M. \& Allen, E. B. 2000 Shifts in arbuscular mycorrihizal communities along an anthropogenic nitrogen deposition gradient. Ecol. Applications 10, 484496.

Eom, A.-H., Hartnett, D. C. \& Wilson, G. W. T. 2000 Host plant species effects on arbuscular mycorrhizal fungal communities in tallgrass prairie. Oecologia 122, 435-444.

Fitter, A. H. \& Moyersoen, B. 1996 Evolutionary trends in root-microbe symbioses. Phil. Trans. R. Soc. Lond. B 351, 1367-1375.

Helgason, T., Daniell, T. J., Husband, R., Fitter, A. H. \& Young, J. P. W. 1998 Ploughing up the wood-wide web? Nature 394, 431.

Hibbett, D. S., Gilbert, L.-B. \& Donoghue, M. J. 2000 Evolutionary instability of ectomycorrhizal symbioses in basidiomycetes. Nature 407, 506-508.

Johnson, N. C., Pfleger, F. L., Crookston, R. K., Simmons, S. R. \& Copeland, P. J. 1991 Vesicular arbuscular mycorrihizas respond to corn and soybean cropping history. New Phytologist 117, 657-663.

Johnson, N. C., Tilman, D. \& Wedin, D. 1992 Plant and soil controls on mycorrihizal fungal communities. Ecology 73, 2034-2042.

Kiers, E. T., Lovelock, C. E., Krueger, E. L. \& Herre, E. A. 2000 Differential effects of tropical arbuscular mycorrhizal fungal inocula on root colonization and tree seedling growth: implications for tropical forest diversity. Ecol. Lett. 3, 106-113.

Klironomos, J. N. 2002 Feedback within soil biota contributes to plant rarity and invasiveness in communities. Nature 417 , 67-70.

Levins, R. 1974 Qualitative analysis of partially specified systems. Ann. NY Acad. Sci. 231, 123-138.

Lutzoni, F., Pagel, M. \& Reeb, V. 2001 Major fungal lineages are derived from lichen symbiotic ancestors. Nature $\mathbf{4 1 1}$ 937-940.

Marler, M. J., Zabinski, C. A. \& Callaway, R. M. 1999 Mycorrhizae indirectly enhance competitive effects of an invasive forb on a native bunchgrass. Ecology 80, 1180-1186.

May, R. M. 1974 Stability and complexity in model ecosystems. Princeton University Press.

Mills, K. E. \& Bever, J. D. 1998 Maintenance of diversity within plant communities: soil pathogens as agents of negative feedback. Ecology 79, 1595-1601.

Modjo, H. S. \& Hendrix, J. W. 1986 The mycorrhizal fungus Glomus macrocarpum as a cause of tobacco stunt disease. Phytopathology 76, 688-691.

Packer, A. \& Clay, K. 2000 Soil pathogens and spatial patterns of seedling mortality in a temperate tree. Nature 404, 278-281.

Pringle, A. 2001 Ecology and genetics of arbuscular mycorrhizal fungi. Phd thesis, Duke University, Durham, NC, USA.

Redecker, D., Hijri, M., Dulieu, H. \& Sanders, I. R. 1999 Phylogenetic analysis of a dataset of fungal 5.8S rDNA sequences shows that highly divergent copies of internal transcribed spacers reported from Scutellospora castanea are of ascomycete origin. Fungal Genet. Biol. 28, $238-244$.

Smith, S. E. \& Read, D. J. 1997 Mycorrhizal symbiosis. San Diego, CA: Academic.

Streitwolf-Engel, R., Boller, T., Wiemken, A. \& Sanders, I. R. 1997 Clonal growth traits of two Prunella species are determ- 
ined by co-occurring arbuscular mycorihizal fungi form a calcareous grassland. F. Ecol. 85, 181-191.

Tilman, D. \& Pacala, S. 1993 The maintenance of species richness in plant communities. In Species diversity in ecological communities (ed. R. E. Ricklefs \& D. Schulter), pp. 13-25. University of Chicago Press.

van der Heijden, M. G. A., Klironomos, J. N., Ursic, M., Moutoglis, P., Streitwolf-Engel, R., Boller, T., Wiemken, A. \& Sanders, I. R. 1998 Mycorrhizal fungal diversity determines plant biodiversity, ecosystem variability and productivity. Nature 396, 69-72.
Van der Putten, W. H., Van Dijk, C. \& Peters, B. A. M. 1993 Plant-specific soil-borne diseases contribute to succession in foredune vegetation. Nature 362, 53-56.

Walley, F. L. \& Germida, J. J. 1996 Failure to decontaminate Glomus clarum NT4 spores is due to spore wall-associated bacteria. Mycorrhiza 6, 43-49.

As this paper exceeds the maximum length normally permitted, the author has agreed to contribute to production costs. 